
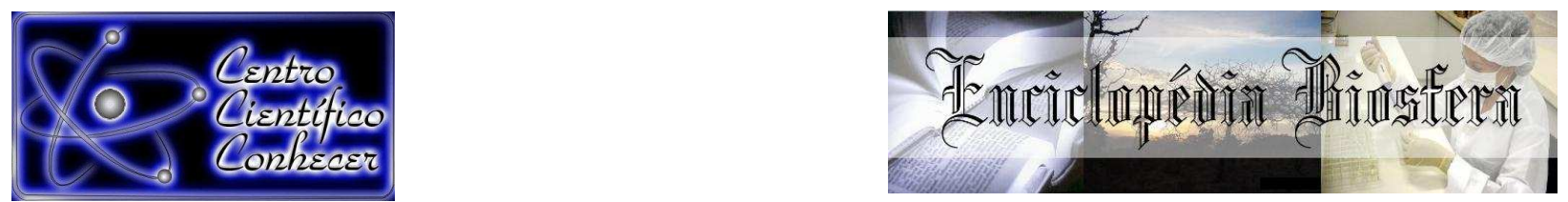

\title{
CLASSIFICAÇÃO DE RISCO DE IDOSOS ATENDIDOS EM UMA UNIDADE DE URGÊNCIA E EMERGÊNCIA
}

\section{Emanuele Caroline da Silva ${ }^{1}$; Vinicius Ventura Lazarini ${ }^{2}$; Diógenes Aparício} Garcia Cortez $^{3}$; Lucia Elaine Ranieri Cortez ${ }^{4}$

1 Acadêmica do curso de Medicina do Centro Universitário de Maringá -

UNICESUMAR - Maringá - Paraná. manu_oie@hotmail.com.br

2 Acadêmico do curso de Medicina do Centro Universitário de Maringá -

UNICESUMAR - Maringá - Paraná.

3 Professor PhD do Mestrado em Promoção da Saúde no Centro Universitário de Maringá- UniCesumar, Maringá, PR Brasil

4 Professora Doutora do Curso de Medicina e Mestrado em Promoção da Saúde no

Centro Universitário de Maringá- UniCesumar, Maringá, PR Brasil

Recebido em: 08/04/2016 - Aprovado em: 30/05/2016 - Publicado em: 20/06/2016

DOI: 10.18677/Enciclopedia Biosfera 2016131

\begin{abstract}
O número de idosos é crescente, e o envelhecimento deixa os indivíduos mais propensos a inúmeros problemas de saúde. O objetivo deste estudo foi analisar os atendimentos de idosos em Unidade de Pronto Atendimento (UPA) com classificação de risco. Foram analisados 1376 prontuários de pacientes idosos atendidos na UPA, quanto a idade do paciente, sexo, diagnóstico - de acordo com a divisão da Classificação Internacional de Doenças (CID 10), além da cor da cadeira no momento da triagem - utilizando a Classificação de Manchester. Os resultados indicaram que as patologias que mais acometeram os idosos estão presentes nos capítulos XVIII (sintomas, sinais e achados anormais de exames clínicos e de laboratório não classificados em outras partes), XIII (doenças do sistema osteomuscular e do tecido conjuntivo ) e IX (doenças do sistema circulatório). O sexo feminino foi o mais acometido, evidenciando uma menor procura de atendimento médico por homens. As cores das cadeiras variaram dentro de cada capítulo do CID, e a coloração amarela (que necessita de rápido atendimento) foi a que mais relacionada com problemas do CID IX - doenças do aparelho circulatório, justificando esta classificação de risco, já que patologias do aparelho circulatório são as que mais causam problemas graves em idosos.
\end{abstract}

PALAVRAS- CHAVE: Idosos, Classificação Internacional de Doenças, Grupos de risco.

\section{RISK RATING OF THE ELDERLY ASSISTED AT AN EMERGENCY AND URGENT CARE UNIT}

\section{ABSTRACT}

The number of elderly people is increasing, and aging makes individuals more prone to health issues. The aim of this study was to analyze the assistance to the elderly at an Emergency Care Unit with risk rating. The analysis consists of 1376 medical 
records of elderly patients assisted at the Emergency Care Unit - the researched data included patient's age, gender, and diagnosis - according to the rank by the International Classification of Diseases (ICD-10), in addition to the color of the seat at the time of screening - using the Manchester Classification. The results pointed that the most affecting pathologies in the elderly were the ones represented on chapters XVIII (symptoms, signals and abnormal findings of clinical and laboratorial exams not classified in other parts), XIII (diseases of the osteomuscular system and connective tissue) and IX (circulatory system diseases). The female gender was the most affected, evincing a lower demand of medical assistance by men. The color of the seats varied among each ICD chapter, in which the color yellow (which needs quick assistance) was the one most related with problems from ICD IX - circulatory system diseases - that risk classification is justified by the fact that pathologies of the circulatory system are those that cause most major problems in elderly people.

KEYWORDS: elderly population, International Classification of Diseases, risk groups.

\section{INTRODUÇÃO}

O número de idosos no mundo está em ascendência, visto que a alteração na estrutura da pirâmide etária é, hoje, uma realidade. O alargamento do ápice da pirâmide mostra uma transição demográfica e evidencia que a expectativa de vida aumentou. Essa transformação torna-se desafio para os cuidados na saúde, necessitando políticas sociais da família, do Estado e também da sociedade (KUCHEMANN, 2012).

Houve também, nos últimos anos, uma transição epidemiológica que contribuiu para o acréscimo de idosos no Brasil (BRASIL, 2012). A ampliação da prevalência das doenças crônicas não transmissíveis (DCNT) caracteriza-se como marcadora do processo de transição epidemiológica do país (CAMPOLINA et al., 2013).

No ano de 1960, havia 3 milhões de idosos no Brasil; em 1975, esse número já era de 7 milhões; em 2008, 20 milhões (VERAS, 2009). No ano de 2012, constatou-se no Brasil aproximadamente 21 milhões de pessoas com idade igual ou superior a 60 anos - o que corresponde a aproximadamente $10 \%$ da população (BRASIL, 2012). Se a previsão dos estudos estiver correta, acredita-se que no ano de 2025 se tenha 32 milhões de pessoas com 60 anos ou mais, tornando- se assim o sexto país com maior número de idosos (BODSTEIN et al., 2014).

Num contexto amplo, o envelhecimento está associado a um maior número de casos de doenças crônicas não transmissíveis - independentes de fatores culturais, sociais e étnicos (GOTTLIEB, 2011), tornando o indivíduo vulnerável, mais propenso a quedas, inaptidões, internações, e óbitos (LANA, 2014). Estimativas nacionais indicam que as DCNT compõem aproximadamente $66,3 \%$ das patologias atuais (CAMPOLINA, et al, 2013). Dentre elas, a hipertensão arterial sistêmica (HAS) é uma das doenças que mais acomete o idoso (VI Diretrizes Brasileiras de Hipertensão Arterial, 2010). Ela tem como principais fatores de risco 0 envelhecimento, obesidade, tabagismo, etilismo, cor de pele negra e escolaridade (ANDRADE et al., 2015).

Diante do aumento das doenças osteoarticulares após os 60 anos de idade, o comprometimento da mobilidade também é característica do envelhecimento, principalmente quando o aumento da idade cronológica é acompanhado pelo sedentarismo e o desuso do aparelho osteomuscular, sendo evidenciada pelo grande número de idosos com lombalgia, variando de $13 \%$ a $49 \%$ (SILVEIRA et al., 2010). A dor lombar aumenta progressivamente com a idade e é de grande 
importância na repercussão na qualidade de vida dos indivíduos acima de 65 anos, fazendo com que 0 atendimento hospitalar seja, muitas vezes, necessário (TAVARES \& LOPES, 2015).

As Unidades de Pronto Atendimento fazem parte da Rede de Atenção às Urgências (BRASIL, 2011) e de acordo com o Ministério da Saúde, são estruturas de complexidade intermediária entre as Unidades Básicas de Saúde e as portas de urgência hospitalares. São integrantes do componente pré-hospitalar fixo e devem ser implantadas em locais/unidades estratégicas para a configuração das redes de atenção à urgência, com acolhimento e classificação de risco em todas as unidades, em conformidade com a Política Nacional de Atenção às Urgências (BRASIL, 2003).

Para atender a demanda de urgências e emergências, a UPA é uma das prioridades do Ministério da Saúde na tentativa de melhorias neste atendimento, com o objetivo de diminuir a superlotação das portas de urgência dos grandes hospitais e também estruturar e organizar a rede de Urgência e Emergência no país (BRASIL, 2003).

Para que haja organização, a Unidade utiliza o Protocolo de Triagem de Manchester (PTM), que, através das cores, classifica os pacientes de nível 1 (vermelho) ao nível 5 (azul), em que o nível 1 é o mais urgente, devendo ser prontamente atendido, e o nível 5 é o menos urgente. Os triados como azuis obedecem à ordem de chegada, visto que nenhum deles possui risco de vida, podendo levar até 4 horas para atendimento. A finalidade do PTM é de melhorar o atendimento aos usuários, dando atenção e suporte imediato às emergências e tornando o fluxo do atendimento o mais eficaz possível (FILHO, 2013).

Este estudo tem como objetivo analisar e descrever as patologias de idosos atendidos em uma unidade de pronto atendimento de acordo com o CID, e verificar a classificação de risco de acordo com as patologias mais prevalentes segundo o CID

\section{MATERIAL E MÉTODOS}

Trata-se de uma pesquisa exploratória e descritiva com aprovação no Comitê de Ética do Unicesumar, através do n527.00 3, em 21 de fevereiro de 2014. A área de estudo é o município de Maringá, localizado na região norte do estado do Paraná, com 367.410 habitantes e uma população idosa de 44.628 indivíduos (BRASIL, 2012).

O levantamento de dados ocorreu na Unidade de Pronto Atendimento Zona Norte do município, através da análise manual de todos os prontuários de atendimento da população idosa (acima de 60 anos), no período de $1^{\circ}$ de Janeiro a 31 de Janeiro de 2014. A Unidade em questão atende a população 24 horas por dia, com livre acesso ou encaminhamento de outros serviços da região.

Foram analisados 1376 prontuários, e as variáveis analisadas foram sexo, idade, diagnóstico médico, de acordo com a Classificação Internacional de Doenças (CID 10), e classificação de risco, segundo PTM. O Protocolo de Triagem de Manchester utiliza uma metodologia em que se divide o atendimento em cinco categorias. Para cada categoria, é atribuída uma cor e um tempo-alvo para o atendimento: nível 1 (emergente, vermelho, atendimento imediato), nível 2 (muito urgente, laranja, 10 minutos), nível 3 (urgente, amarelo, 60 minutos), nível 4 (pouco urgente, verde, 120 minutos) e nível 5 (não urgente, azul, 240 minutos) (FILHO, 2013).

Foram detalhados os três grupos numericamente mais relevantes, considerando o CID, sendo: Capítulo XVIII (sintomas, sinais e achados anormais de 
exames clínicos e de laboratório não classificados em outras partes), Capítulo XIII (relacionado às doenças do sistema osteomuscular e do tecido conjuntivo) e Capítulo IX (que representa doenças do sistema circulatório).

Os dados foram analisados mediante estatística descritiva, utilizando-se as distribuições de frequências absoluta e relativa. A análise foi realizada por meio do Excel versão 2010.

\section{RESULTADOS E DISCUSSÃO}

A tabela 1 apresenta a ocorrência de atendimentos segundo CID. Houve o predomínio dos capítulos XVIII, XIII e IX - sendo que o capítulo XVIII $(19,23 \%, n=$ 264) representa os sintomas, sinais e achados anormais de exames clínicos e de laboratório não classificados em outras partes. O capítulo XIII relaciona-se às doenças do sistema osteomuscular e do tecido conjuntivo. E, por fim, o capítulo IX representa doenças do sistema circulatório.

Ressaltam-se em menor ocorrência os Capítulos XI $(7,87 \%, n=108)$ e $X$ $(7,28 \%, n=100)$, que representam as doenças do aparelho digestivo e doenças do aparelho respiratório, respectivamente.

TABELA 1 - Atendimento dos pacientes de acordo com a Classificação Internacional das Doenças (CID). Maringá, 2014.

\begin{tabular}{ccc}
\hline CID & Quantidade (n) & $\%$ \\
I & 70 & 5,10 \\
III & 7 & 0,51 \\
IV & 41 & 2,99 \\
V & 31 & 2,26 \\
VI & 26 & 1,89 \\
VII & 9 & 0,66 \\
VIII & 36 & 2,62 \\
IX & 148 & 10,75 \\
X & 100 & 7,28 \\
XI & 108 & 7,87 \\
XII & 48 & 3,50 \\
XIII & 259 & 18,82 \\
XIV & 79 & 5,75 \\
XVIII & 253 & 18,38 \\
XIX & 91 & 6,63 \\
XX & 38 & 2,77 \\
XXI & 21 & 1,53 \\
\hline
\end{tabular}

Fonte: dados da pesquisa.

Os capítulos XVIII, XIII e IX corresponderam a aproximadamente $50 \%$ de todos os prontuários de idosos analisados na UPA, evidenciando a importância do aprofundamento no assunto.

O CID XVIII representou 18,38\% $(n=253)$ dos prontuários verificados e este indica achados anormais nos exames clínicos, sem que haja classificação específica em outros capítulos do CID. Esse número é bem representativo, sendo importante ressaltar que o envelhecimento é um processo multifatorial, em que há alterações de caráter químico, biológico, funcional, cultural, social e até mesmo ambiental (SANTOS et al., 2009). Dessa forma, por alterar os indivíduos em todos os parâmetros, achados anormais no exame físico tornam-se mais frequentes. Tais 
achados incluem tosse, mal estar geral, dor torácica, sem que se conheça o real fator desencadeante do processo.

As doenças do sistema osteomuscular e do tecido conjuntivo estavam presentes em $18,82 \%(n=259)$ dos prontuários analisados. Elas acometem em grande proporção a terceira idade, já que nesse período ocorrem alterações no organismo, em especial no sistema locomotor - a sarcopenia - que pode ser causada por diversos mecanismos, de deficiência na síntese protéica às neoplasias (FREITAS et al., 2015). A qualidade de vida dos idosos é influenciada pela redução e eficácia do sistema muscular, podendo torná-los mais dependentes de cuidado e atenção (PÍCOLI et al., 2011).

A prática de exercícios físicos pode auxiliar a diminuir patologias relacionadas ao sistema osteomuscular e conjuntivo, oferecendo ao idoso uma estabilidade corporal, equilíbrio na postura e marcha, tornando-o mais ativo e prevenindo-o de quedas secundárias ao enfraquecimento muscular, comum nessa idade (PEDRINELLI et al., 2009).

De acordo com a literatura científica, as doenças crônicas - mais comuns na senilidade - danificam a capacidade funcional dos idosos, constituindo fatores importantes a serem considerados pela sociedade em geral, já que a debilidade os fará mais vulneráveis. Entre os fatores mais agravantes encontra-se a incapacidade funcional, impedindo-os de realizar atividades comuns do dia-dia, conhecidas como atividades básicas de vida diária (ABVD), tornando-os dependentes de cuidados extras e com menos qualidade de vida (FIALHO, 2014).

Verificou-se também que $10,75 \% \quad(n=148)$ dos atendimentos são classificados segundo Capítulo IX, os quais caracterizam por distúrbios circulatórios. As doenças cardiovasculares constituem a principal causa de mortalidade no mundo todo, abrangendo aproximadamente $30 \%$ dos casos (MENDIS et al., 2011). Em estudos realizados em 2013, 13,9\% $(n=315)$ dos pacientes idosos analisados na UPA de São José dos Campos foram diagnosticados com doenças cardiovasculares (ZEPKA \& CAETANO, 2014).

A hipertensão arterial sistêmica (HAS) constitui o principal fator de risco para as doenças cardiovasculares, e acomete de $51,8 \%$ a $61,5 \%$ dos idosos (ZATTAR, 2013). De acordo com as VI diretrizes brasileiras de hipertensão, de 2010, as relações entre HAS e idade são evidentes, sendo que há uma prevalência de aproximadamente $60 \%$ dessa patologia em indivíduos acima de 65 anos. É considerada precursora do acidente vascular encefálico e do infarto agudo do miocárdio (IAM), além de possivelmente tornar, aos poucos, um indivíduo hígido, dependente (LOPES \& MARCON, 2013), necessitando, portanto, grande atenção médica.

Ademais, as doenças do aparelho circulatório foram responsáveis por $37,17 \%$ dos óbitos relacionados aos idosos no período de 2006 a 2010 (PIUVEZAM et al., 2015). Assim como em muitas outras condições crônicas inerentes ao envelhecimento, as doenças do aparelho circulatório possuem grande impacto na mortalidade, principalmente quando associadas ao tabagismo, sedentarismo, dieta inapropriada, consumo excessivo de álcool, ou pela concomitância com diabetes mellitus, hipertensão arterial ou desordens genéticas (VERAS, 2011). 
Capítulo IX

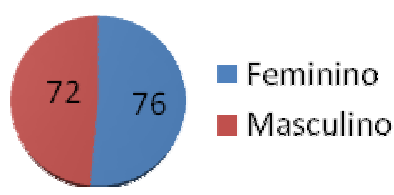

Capítulo XIII

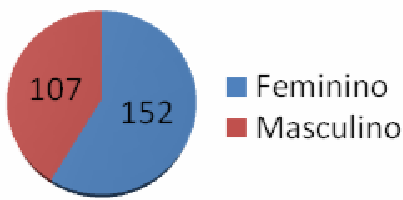

Capítulo XVIII

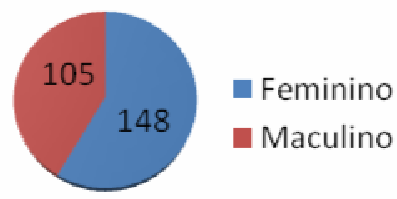

GRÁFICO 1 - Atendimentos classificados nos capítulos IX, XIII e XVIII de acordo com o sexo

O gráfico 1 relaciona o sexo com os capítulos do CID mais freqüentes do presente estudo. Verificou-se maior ocorrência entre as mulheres, nos capítulos IX $(51,4 \%, n=76)$, XIII $(58,8 \%, n=152)$ e XVIII $(58,7 \%, n=148)$.

Estudo feito em um ambulatório de geriatria no Distrito Federal (LINHARES, 2003), constatou que $70 \%$ dos pacientes eram do sexo feminino. BORGES (2013) acredita que o fato de os homens procurarem os serviços de atendimento mais tardiamente, pode fazer com que tenham prognósticos piores, levando a tratamentos de difíceis resoluções, além de mais dispendiosos.

A supervalorização do sexo masculino pela própria sociedade pode ser responsável por tornar o homem menos cuidadoso com a sua saúde, procurando em menor quantidade os serviços de atendimento médico. Além disso, o fato de as Unidades Básicas de Saúde possuírem grande quantidade de mulheres pode gerar no homem a impressão de serem feminilizadas, evitando a procura pelo atendimento (VIEIRA et al., 2013). ZEPKA \& CAETANO (2014) confirmaram, através de pesquisas, que a demanda masculina pelo atendimento médico é menor também no serviço de urgência e emergência.

No ano de 2009 foi instituído pelo Ministério da Saúde a Política Nacional de Atenção Integral à Saúde do Homem (PNAISH). A inserção do PNAISH envolveu uma equipe multidisciplinar, de gestores às equipes técnicas (LEAL et al., 2012). São cinco focos especiais: Acesso e Acolhimento, Saúde Sexual e Reprodutiva, Paternidade e Cuidado, Doenças prevalentes na população masculina e Prevenção de Violências e Acidentes (BRASIL, 2009). Esses eixos visam inserir o homem nos cuidados à própria saúde, estimulando-os a procurarem, com mais intensidade, o atendimento pré-hospitalar e hospitalar.

TABELA 2 - Classificação Internacional de Doenças (CID) de acordo com a faixa etária. Maringá, 2014.

Faixa Etária

CID

\begin{tabular}{cccc}
\hline & Capítulo IX & Capítulo XIII & Capítulo XVIII \\
\hline $60-64$ & $40(27,0 \%)$ & $93(35,9 \%)$ & $64(25,2 \%)$ \\
$65-69$ & $29(19,5 \%)$ & $53(20,4 \%)$ & $60(23,7 \%)$ \\
$70-74$ & $30(20,2 \%)$ & $41(15,8 \%)$ & $43(16,9 \%)$ \\
$75-79$ & $28(18,9 \%)$ & $35(13,5 \%)$ & $38(15,0 \%)$ \\
$80-85$ & $21(14,1 \%)$ & $23(8,8 \%)$ & $32(12,6 \%)$ \\
$>85$ & $0(0,0 \%)$ & $14(5,4 \%)$ & $16(6,3 \%)$ \\
TOTAL & $148(100 \%)$ & $259(100 \%)$ & $253(100 \%)$ \\
\hline
\end{tabular}

Fonte: dados da pesquisa. 
A tabela 2 apresenta as faixas etárias, segundo os capítulos do CID mais ocorrentes (IX, XIII e XVIII). Nos três segmentos do CID é observado um maior número de atendimentos entre 60 a 64 anos $(n=197)$, seguido da faixa etária de 65 a 69 anos $(n=142)$, e menor número de atendimentos entre aqueles com acima de 85 anos de idade ou mais $(n=30)$.

O maior atendimento aos idosos na faixa etária de 60-64 referiu-se ao CID $\mathrm{XIII}$, relacionado às doenças do sistema osteomuscular e do tecido conjuntivo.

No entanto, nas faixas etárias de 65 - 69; 70 - 74, 75 - 79, 80 - 85 e maior que 85 anos, o maior número de atendimento se relaciona ao CID XVIII que aborda o capítulo de sintomas, sinais e achados anormais de exames clínicos e de laboratório não classificados em outras partes.

TABELA 3: Classificação de risco, de acordo com a classificação de Manchester, dos atendimentos a idosos em UPA Zona Norte de Maringá de acordo com o CID -. Maringá, 2014.

\begin{tabular}{cccc}
\hline & IX & XIII & XVIII \\
\cline { 2 - 4 } Amarela & & & \\
Azul & $74,6 \%$ & $25,2 \%$ & $42,8 \%$ \\
Verde & 0 & $1,1 \%$ & $0,38 \%$ \\
\hline
\end{tabular}

Fonte: dados da pesquisa.

A sobrevida do paciente crítico é dependente do tratamento inicial, imediato e competente (OREDSSON et al., 2011). A Superlotação nos Serviços de Emergências Hospitalar (SEH) gera uma lentificação no fluxo dos atendimentos e acaba por influenciar a equipe atendente, podendo prejudicar a qualidade do acolhimento (BITTENCOURT, 2009). Assim, evidencia-se que a eficiência da rede de urgência e emergência é dependente de uma triagem de qualidade.

Diante disso, conforme tabela 3 , os capítulos do CID também foram subdivididos de acordo com a classificação de risco do paciente no momento da triagem, pela equipe da enfermagem. Dessa maneira é possível analisar se a procura pelo serviço de atendimento era realmente necessária.

A cadeira de coloração azul, conforme já exposto, é classificada como a de menor preocupação, em que o atendimento deve ser feito pela ordem de chegada, já que não há urgência e/ou emergência. Nos três capítulos analisados, evidencia-se que a procura pelo atendimento com a cor azul foi quase nula. Isso é sinal de que os usuários não estão procurando 0 atendimento urgente sem que haja real necessidade, pois o nível de complexidade das patologias na coloração azul é pacífico de ser solucionado em Unidade Básica de Saúde.

A coloração amarela, mais freqüente entre os idosos com patologias classificadas pelo CID IX (com 74,6\%), diz respeito aos usuários críticos e semi críticos, que necessitam de atendimento o mais rápido possível e é relacionado com as doenças do sistema circulatório.

No capítulo XVIII, $42,8 \%$ dos atendimentos também foram classificados pela triagem como amarelo, mostrando que os sintomas, sinais e achados anormais de exames clínicos e de laboratório não classificados em outras partes também possuem relativa urgência no atendimento, ainda que $56,82 \%$ desse capítulo correspondam à coloração verde, evidenciando que tais sinais e achados pode ter resolução mais facilitada que o capítulo IX. 
A cor verde sinaliza situações pouco urgentes, em que o paciente pode aguardar até 120 minutos para receber atendimento, pois a situação em que ele se encontra não o expõe ao risco de morte. As doenças do sistema osteomuscular e do tecido conjuntivo, representadas pelo CID XIII (73,7\%), foram as mais contabilizadas nessa coloração.

A principal consequência das patologias relacionadas ao CID XIII é a incapacidade funcional do indivíduo que, apesar de trazer desconforto e impedi-lo de realizar atividades que fazem parte do seu cotidiano, raramente podem cursar com quadro clínico que pode levá-lo a morte de maneira imediata.

\section{CONCLUSÃO}

A relevância deste estudo é de conhecer o perfil do idoso que busca o pronto atendimento, quantificando a idade e analisando o sexo para relacionar possíveis patologias com faixas etárias e gêneros, possibilitando a prevenção e resolução das doenças. Entretanto, tornou-se limitado, visto que poucos estudos foram realizados utilizando-se dos capítulos do CID aqui empregados.

Os dados aqui apresentados demonstram que a maior demanda pelo atendimento de idosos na Unidade de Pronto Atendimento da Zona Norte da cidade de Maringá, estão relacionadas aos Capítulos XVIII, Capítulo XIII e Capítulo IX do CID. Idosos do sexo feminino e na faixa etária entre 60 e 64 anos foram as mais acometidas pelas patologias dos capítulos referidos.

Há poucos estudos que comparam o atendimento do idoso com a Classificação de triagem de Manchester, dificultando o cruzamento de dados. Porém, na UPA da cidade de Maringá, evidencia-se que os atendimentos realizados às patologias com diagnóstico pertencente ao Capítulo IX, dentre os elencados, são os mais agravantes nos idosos, uma vez que a triagem amarela representa que 0 atendimento deve ser rápido, diferentemente dos Capítulos XIII e XVIII que, por serem menos ofensivos à saúde do paciente, podem esperar mais tempo para atendimento, sendo representados, em maior parte, pela cor verde.

A quase ausência de atendimentos de cor azul, nos três capítulos analisados, corrobora para o fato de que os idosos não estão procurando a Unidade de Pronto Atendimento sem a real necessidade. Uma das justificativas para isso pode ser a funcionalidade do Programa de Saúde da Família no local estudado, promovendo a busca ativa e o acompanhamento dos pacientes nas suas áreas de atuação, realizando promoção e prevenção da saúde dos idosos.

\section{REFERÊNCIAS}

ANDRADE, S. S. A; STOPA, S. R; BRITO, A. S; CHUERI, P. S; SZWARCWALD, C. L; MALTA, D. C. Prevalência de hipertensão arterial autorreferida na população brasileira: análise da Pesquisa Nacional de Saúde, 2013. Epidemiol. Serv. Saúde v.24 n.2, Brasília, $2015 . \quad$ Disponível em: <http://www.scielosp.org/pdf/ress/v24n2/2237-9622-ress-24-02-00297.pdf>. Doi: 10.5123/S1679-49742015000200012. Acesso em: 23 set. 2015.

BITTENCOURT, R. J; HORTALE, V. A. Intervenções para solucionar a superlotação nos serviços de emergência hospitalar: uma revisão sistemática. Cad. Saúde Pública, Rio de Janeiro, 25(7):1439-1454, jul, 2009. Disponível em: <http://www.scielosp.org/pdf/csp/v25n7/02.pdf>. Acesso em: 15 set. 2015. 
BORGES, L. M; SEIDL, E. M. F. Efeitos de intervenção psicoeducativa sobre a utilização de serviços de saúde por homens idosos. Interface (Botucatu), v.17, n.47, <http://www.scielo.br/pdf/icse/v17n47/aop4013.pdf>. Doi: 10.1590/S141432832013005000024. Acesso em: 15 out. 2015.

BODSTEIN, A; LIMA, V. V. A; BARROS, A. M. A. A vulnerabilidade do idoso em situações de desastres: necessidade de uma política de resiliência eficaz. Ambiente \& Sociedade. São Paulo v. XVII, n. 2 n p. 157-174 n abr.-jun. 2014. Disponível em: <http://www.scielo.br/pdf/asoc/v17n2/a11v17n2.pdf>. Acesso em: 12 jul. 2015.

BRASIL. Ministério da Saúde. Portaria n¹863/GM 29 de setembro de 2003. Institui a Política Nacional de Atenção às Urgências. Brasilia, DF. Disponível em: $<\mathrm{http}$ ://bvsms.saude.gov.br/bvs/publicacoes/politica_nac_urgencias.pdf>. Acesso em: 03 jul. 2015.

BRASIL. Ministério da Saúde. Portaria n¹600 07 de julho de 2011. Reformula a Política Nacional de Atenção as Urgências no Sistema Único de Saúde (SUS). Diário Oficial da União, Brasília, DF, 08, jul. 2011. Disponível em: <http://bvsms.saude.gov.br/bvs/saudelegis/gm/2011/prt1600_07_07_2011.html>. Acesso em: 09 jul. 2015.

BRASIL - 2012: Indicadores e dados básicos. Disponível em: <http://tabnet.datasus.gov.br/cgi/deftohtm.exe?ibge/cnv/popbr.def>. Acesso em: 12 jul. 2015.

BRASIL - 2012: Indicadores e dados básicos. Disponível em: < http://tabnet.datasus.gov.br/cgi/tabcgi.exe?ibge/cnv/popbr.def>. Acesso em: 18 jul. 2015.

BRASIL. Ministério da Saúde. Portaria n¹.944, de 27 de Agosto de 2009. Institui no âmbito do Sistema Único de Saúde (SUS), a Política Nacional de Atenção Integral à Saúde do Homem. Disponível em: <http://bvsms.saude.gov.br/bvs/saudelegis/gm/2009/prt1944_27_08_2009.html>.

Acesso em: 12 mar. 2015.

BRASIL. Ministério da Saúde. Epidemiologia e Serviços de Saúde. Epidemiol. Serv. Saúde, Brasília, 21(4):529-532, out-dez 2012. Disponível em: $<$ http://bvsms.saude.gov.br/bvs/periodicos/epidemiologia_servicos_saude_volume21 _n4.pdf>. Acesso em: 21 jun. 2015

CAMPOLINA, A. G; ADAMI, F; SANTOS, J. L. F; LEBRÃO, M. L. A transição de saúde e as mudanças na expectativa de vida saudável da população idosa: possíveis impactos da prevenção de doenças crônicas. Cad. Saúde Pública, Rio de Janeiro, 29(6):1217-1229, jun, 2013. Disponível em: <http://www.scielosp.org/pdf/csp/v29n6/a18v29n6.pdf>. Acesso em: 29 nov. 2015

DIRETRIZES BRASILEIRAS DE HIPERTENSÃO VI. Revista Hipertensão, v.13, n.1. jan./mar. 2010. Disponível em: <http://publicacoes.cardiol.br/consenso/2010/Diretriz_hipertensao_associados.pdf>. 
Acesso em: 02 fev. 2016.

FIALHO, C. B; COSTA, M. F. L; GIACOMIN, K. C; FILHO, A. I. L. Capacidade funcional e uso de serviços de saúde por idosos da Região Metropolitana de Belo Horizonte, Minas Gerais, Brasil: um estudo de base populacional. Cad. Saúde Pública, Rio de Janeiro, 30(3):599-610, mar, 2014. Disponível em: <http://www.scielosp.org/pdf/csp/v30n3/0102-311X-csp-30-3-0599.pdf>. Acesso em: 23 out. 2015

FILHO, L. A. M. S; Revisão sistemática do Sistema de Triagem de Manchester na estratificação de risco. Salvador (Bahia) Junho, 2013. Disponível em: <http://gbcr.org.br/downloads>. Acesso em 2 mai. 2016.

FREITAS, A. F; PRADO, M. A; CAÇÃO, J. C; BERETTA, D; ALBERTINI, S. Sarcopenia e estado nutricional de idosos: uma revisão da literatura. Arq. Ciênc. Saúde. 2015 jan-mar; 22(1) 09-13. Disponível em: < http://www.cienciasdasaude.famerp.br/index.php/racs/article/view/19/pdf_2> Acesso em: 4 abr. 2016.

GOTTLIEB, M. G. V; SCHWANKE, C. H. A; GOMES, I; CRUZ, I. B. M. Envelhecimento e Longevidade no Rio Grande do Sul: um perfil histórico, étnico e de morbi-mortalidade dos idosos. Revista brasileira de geriatria e gerontologia. Rio de Janeiro, 2011. Disponível em: <http://www.scielo.br/pdf/rbgg/v14n2/v14n2a16>. Acesso em: 20 mar. 2016.

KUCHEMANN, B. A. Envelhecimento populacional, cuidado e cidadania: velhos dilemas e novos desafios. Soc. estado. vol.27 no.1. Brasília Jan./Apr. 2012. Disponível em: <http://dx.doi.org/10.1590/S0102-69922012000100010>. Acesso em: 02 mar. 2016.

LANA, L. D; SCHNEIDER, R. H. Síndrome de fragilidade no idoso: uma revisão narrativa. Rev. Bras. Geriatr. Gerontol., Rio de Janeiro, 2014. Disponível em: <http://www.scielo.br/pdf/rbgg/v17n3/1809-9823-rbgg-17-03-00673.pdf>. Acesso em: 13 jul. 2015.

LEAL, A. F; FIGUEIREDO, W. S; SILVA, G. S. N. O percurso da Política Nacional de Atenção Integral à Saúde dos Homens (PNAISH), desde a sua formulação até sua implementação nos serviços públicos locais de atenção à saúde. Ciênc. saúde coletiva vol.17 no.10 Rio de Janeiro Oct. 2012. Disponível em: <http://www.scielo.br/pdf/csc/v17n10/10.pdf>. Acesso em: 27 jul. 2015.

LINHARES, C. R. C; COELHO, V. L. D; GUIMARÃES, R. M; CAMPOS, A. P. M; CARVALHO, N. T. Perfil da clientela de um ambulatório de geriatria do Distrito Federal. Psicol. Refl. Crit., v.16, n.2, p.319-26, 2003. Disponível em: <http://www.scielo.br/pdf/prc/v16n2/a12v16n2.pdf>. Acesso em: 19 fev. 2016.

LOPES, M. C. L; MARCON, S. S. A vivência do idoso e sua família com a hipertensão arterial. Cienc Cuid Saude 2013 Abr/Jun; 12(2):241-248. Disponível em: 
<http://www.periodicos.uem.br/ojs/index.php/CiencCuidSaude/article/viewFile/21745/ pdf> Doi: 10.4025/cienccuidsaude.v12i2.21745. Acesso em: 4 mai. 2016

MENDIS, S.; PUSKA, P.; NORRVING, B. Global Atlas on Cardiovascular Disease Prevention and Control. Geneva: World Health Organization, 2011. Disponível em: <http:whqlibdoc.who.int/publications/2011/9789241564373_eng.pdf?ua=1>. Acesso em: 3 abr. 2015.

OREDSSON, S; JONSSON, H; ROGNES, J; LIND, L; GÖRANSSON, K. E; EHRENBERG, A ; ASPLUND, K; CASTRÉN, M; FARROHKNIA, N. A systematic review of triage-related interventions to improve patient flow in emergency departments Scandinavian Journal of Trauma, Resuscitation and Emergency medicine [Internet]. BioMed Central Ltd; 2011 Jan;19(1):43. Disponível em: $<$ http://www.pubmedcentral.nih.gov/articlerender.fcgi?artid=3152510\&tool=pmcentre z\&rendetype=abstract>. Acesso em: 30 de Out. 2015. Acesso em: 09 jul. 2015.

PEDRINELLI, A; LEME, L. E. G; NOBRE, R. S. A. O efeito da atividade física no aparelho locomotor do idoso. Rev. bras. ortop. vol.44 no.2 São Paulo Mar./Apr. $2009 . \quad$ Disponível em: <http://www.scielo.br/pdf/rbort/v44n2/a02v44n2.pdf>. Acesso em: 10 jul. 2015.

PÍCOLI, T. S; FIGUEIREDO, L. L; PATRIZZI, L. J. Sarcopenia e envelhecimento. Fisioter. mov. (Impr.) vol.24 no.3 Curitiba July/Sept. 2011. Disponível em: <http://www.scielo.br/pdf/fm/v24n3/10.pdf>. Acesso em: 20 out. 2015.

PIUVEZAM, G; MEDEIROS, W. R; COSTA, A. V; EMERENCIANO, F. F; SANTOS, R. C; SEABRA, D. S. Mortalidade em Idosos por Doenças Cardiovasculares: Análise Comparativa de Dois Quinquênios. Universidade Federal do Rio Grande do Norte, Natal, RN - Brasil. Arq Bras Cardiol. 2015. Disponível em: <http://www.scielo.br/pdf/abc/2015nahead/pt_0066-782X-abc-20150096.pdf>. Acesso em: 13 mai. 2015.

SANTOS, F. H; ANDRADE, V. M; BUENO, O. F. A. Envelhecimento: um processo multifatorial. Psicologia em Estudo, Maringá, v. 14, n. 1, p. 3-10, jan./mar. 2009. Disponível em: <http://www.scielo.br/pdf/pe/v14n1/a02v14n1.pdf>. Acesso em: 05 ago. 2014.

SILVEIRA, M. M; PASQUALOTTI, A; COLUSSI, E. L; VIDMAR, M. F; WIBELINGER, L. M. Abordagem fisioterápica da dor lombar crônica no idoso.. Revista Brasileira de Ciências da Saúde, ano 8, ํo 25, jul/set 2010. Disponível em: < http://seer.uscs.edu.br/index.php/revista_ciencias_saude/article/view/1062/856> Acesso em: 20 dez. 2015.

TAVARES, R. O; LOPES, R. G. C., Atividade física no envelhecimento, uma contribuição para a qualidade de vida. Revista Portal de Divulgação, n.45, Ano V. São Paulo/SP, Jun/Jul/Ago. 2015.

VERAS, R. Envelhecimento populacional contemporâneo: demandas, desafios e inovações. Rev. Saúde Pública, v.43, n.3, p.548-54, 2009. Disponível em: <http://www.scielo.br/pdf/rsp/v43n3/224.pdf>. Acesso em: 24 jul. 2015. 
VERAS, R. P. Estratégias para o enfrentamento das doenças crônicas: um modelo em que todos ganham. Revista Brasileira de Gerontologia, Rio de Janeiro, 2011. Disponível em: <http://dx.doi.org/10.1590/S1809-98232011000400017>. Acesso em: 2 jun.2015.

VIEIRA, K. L. D; GOMES, V. L. O; BORBA, M. R; COSTA, C. F. S. Atendimento da população masculina em unidade básica saúde da família: motivos para a (não) procura. Esc. Anna Nery v.17 n.1 Rio de Janeiro Jan./Mar. 2013. Disponível em: <http://www.scielo.br/pdf/ean/v17n1/17.pdf>. Acesso em: 14 set. 2015.

ZEPKA, A. P. S; CAETNAO, T. L. A urgência no atendimento ao idoso: estudo de caso. Revista Univap. São José dos Campos-SP-Brasil, v. 21, n. 37, jul.2015. ISSN 2237-1753. Disponível em: http://revista.univap.br/index.php/revistaunivap/article/viewFile/280/267>. Acesso em: 17 jan. 2016

ZATTAR, L. C; BOING, A. F; GIEHL, M. W. C; D'ORSI, E. Prevalência e fatores associados à pressão arterial elevada, seu conhecimento e tratamento em idosos no sul do Brasil. Cad. Saúde Pública, Rio de Janeiro, 29(3):507-521, mar, 2013. Disponível em: <http://www.scielo.br/scielo.php?script=sci_arttext\&pid=S0102311X2013000300009>. Acesso em: 9 dez. 2015 